\title{
Anwendung der Suprasorb CNP-Drainagefolie in der septischen Bauchchirurgie
}

\author{
Carl Meißner, Gerd Meißner
}

\section{Zusammenfassung}

Hintergrund: Infektionen sind in der Chirurgie immer noch ein großes Problem. Die großen Entzündungen im Unterleib, z.B. fibrinöser Art, stellen immer noch eine Herausforderung in der Chirurgie dar. Unserer Meinung nach führte die intraabdominelle Vakuumtherapie zu einer Verbesserung der Infektsituation. Eine Standardmethode der offenen Vakuumtherapie am Abdomen besteht nicht. Wir möchten im folgenden Fallbericht eine mögliche Verbesserung in der Versorgung unserer Patienten aufzeigen. Methoden (Fallbericht): Eine 64-jährige Patientin stellt sich aufgrund progredienter abdomineller Beschwerden in der Notaufnahme vor. Die Patientin wurde einer Laparotomie unterzogen. Auf diese folgten Appendektomie, umfangreiche Abdominallavage und Anlage eines Systems zur Vakuumversiegelung mit CNP-Film. Ergebnisse: Keine Komplikationen postoperativ. Die Patientin wurde am 12. Tag nach der Operation in die Häuslichkeit entlassen. Schlussfolgerungen: In diesem Bericht präsentieren wir eine gelungene Kombination aus einem System zur Vakuumversiegelung und Suprasorb CNP-Drainagefolie.
Use of Suprasorb CNP Drainage Film in Surgery of the Septic Abdomen

Background: Infections are still a major problem in surgery. Massive inflammations in the lower abdomen, e.g., those of a fibrinous nature, remain as a great challenge for the surgeon. In our oipinion, intra-abdominal vacuum therapy leads to an improvement in the infectious situation. However there is no standardised method for open vacuum therapy of the abdomen. In the present case report we want to illustrate possible improvements in the management of our patients. Methods (Case Report): A 64-year-old female patient presented to the emergency department because of progressive abdominal complaints. The patient underwent a laparotomy. This was followed by appendectomy, extensive abdominal lavage and placement of a system for vacuum sealing with CNP film. Results: There were no postoperative complications. The patient was released home on the 12th day after surgery. Conclusion: In this case report we have described the successful combination of a system for vacuum sealing and drainage with Suprasorb CNP film.

\section{Einleitung}

Infektionen sind in der Chirurgie immer noch ein großes Problem. Gründe für eine „offenen Bauch“ sind in einer beabsichtigten offenen Behandlung, einer verzögerten primären Heilung oder Infektionen [1] zu suchen. Die riesige Entzündung im Unterleib, z.B. fibrinöser

OP-JOURNAL 2013; 29: 267-268

(c) Georg Thieme Verlag KG Stuttgart · New York DOI http://dx.doi.org/10.1055/s-0033-1360151

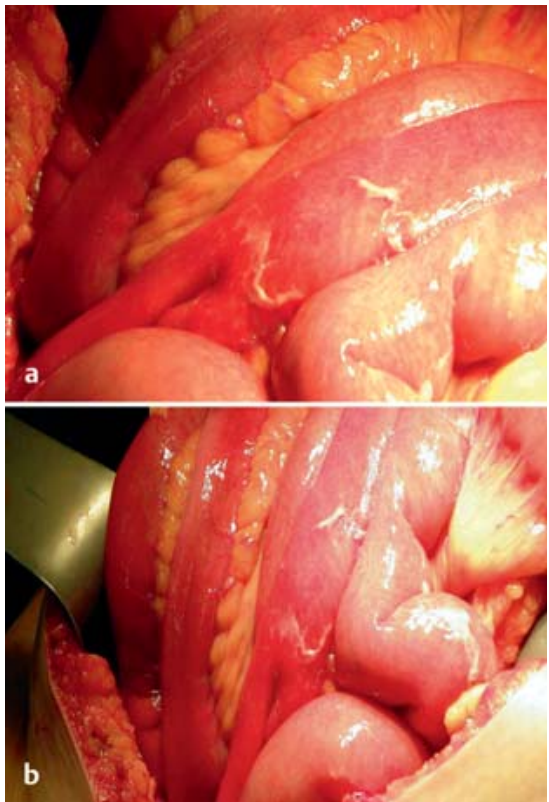

Abb. $1 \mathbf{a}$ und $\mathbf{b}$ Fibrinöse Peritonitis bei Appendicitis perforata und einem MPI („Mannheimer Peritonitis Index“) von 29. (Abb. 2 und 3).

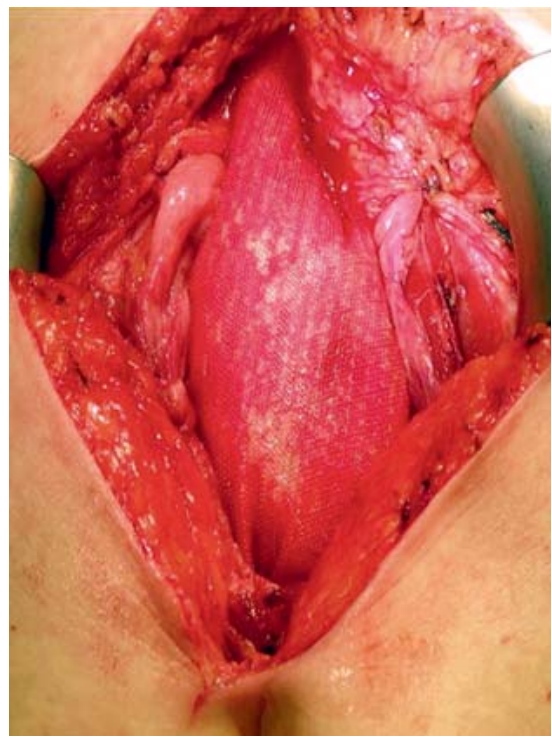

Abb. 2 Einlage der CNP-Drainagefolie.
Art, ist eine Herausforderung in der Chirurgie. Die Anwendung einer abdominellen Vakuumtherapie führte unserer Meinung nach zu zu einer Verbesserung.

Wir berichten über einen Fall von Appendicitis perforata mit Peritonitis, gefolgt durch Laparotomie, Appendektomie und Anwendung des Suprasorb CNP-Films mit Vakuumversiegelung. 


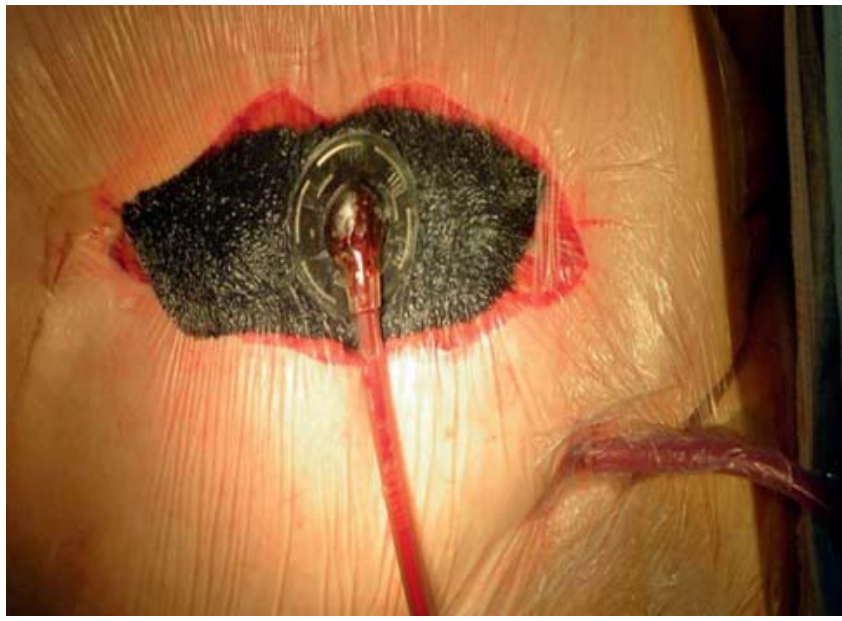

Abb. 3 Einlage der CNP-Drainagefolie und der Vakuumversiegelung.

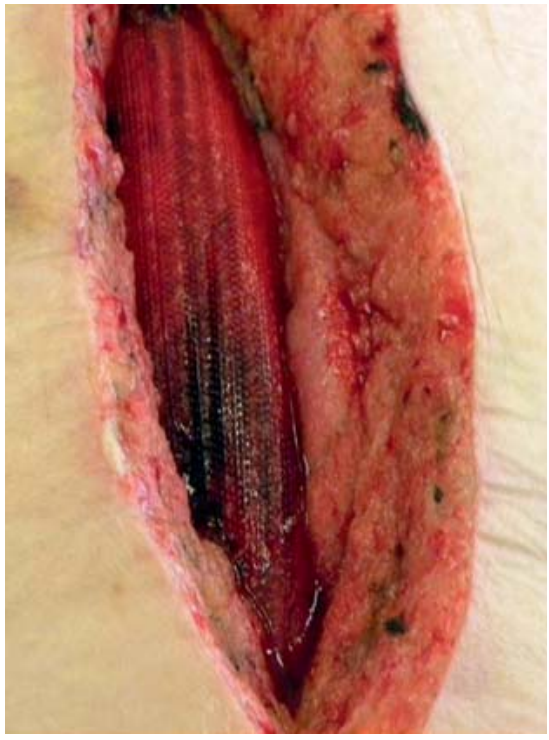

Abb. 4 VAC-(Vacuum-Assisted Closure-)Entfernung und Sekundärnaht der Bauchdecke.

\section{Fallbericht}

Die Patientin stellte sich in der Nacht vom 21.8. zum 22.8. wegen abdomineller Beschwerden in unserer Notfallambulanz vor. Anamnestisch ließen sich Schmerzen seit dem 20.8. eruieren. Am Morgen des 22.8. war ein deutlicher Druckschmerz im rechten Unterbauch und ein Perkussionsschmerz rechts und links nachweisbar. Sonografisch ließ sich Flüssigkeit in der Abdominalhöhle feststellen. Wir stellten die Verdachtsdiagnose einer akuten Appendizitis und unverzüglich war die Indikation zur Laparotomie gegeben.

\section{Laparotomie vom 22.8}

Intraoperativ fand sich eine Appendicitis perforata mit Peritonitis. Es folgten die Appendektomie, ausgedehnte Abdominallavage und die Anlage eines Systems zur Vakuumversiegelung mit CNP-Drainagefolie (Abb. 1 a und b).

Es folgten Operationen am 25.8. und 28.8., bis schließlich das System zur Vakuumversiegelung entfernt werden konnte (Abb. 4).

Am 3.9. konnte die Patientin bei subjektivem Wohlbefinden und reizlosen Wundverhältnissen in die Häuslichkeit entlassen werden. Die ambulante Abschlusskonsultation am 24.9. war ohne pathologisches Korrelat und regelrecht.

\section{Diskussion}

Ein standardisiertes Verfahren am offenen Abdomen gibt es nicht, jedoch wollen wir zeigen, dass Entwicklungen uns
Hilfestellungen zur Versorgung unserer Patienten geben. In den letzten Jahren stellt temporär die Vakuumversiegelung eine gute Versorgungsmöglichkeit des „Open Abdomen“ bis zum terminalen Verschluss des Abdomens dar [2]. Der Einsatz des CNP-Films in dem Verfahren der septischen Chirurgie, insbesondere am „offenen Abdomen“, hat klare Vorteile: schneller Verschluss der Bauchwand, gute Mobilisierung des Patienten, Reduktion von Ödemen und verbesserte Entfernung von postoperativen Seromen [3]. Es ist sehr einfach einzusetzen.

\section{Literatur}

1 Bovill E, Banwell PE, Teot L et al.; International Advisory Panel on Topical Negative Pressure Topical negative pressure wound therapy: a review of its role and guidelines for its use in the management of acute wounds. Int Wound J 2008; 5: 511-529

2 Wild T, Otto F, Mojarrad L et al. [Vacuum therapy-basics, indication, contraindication and cost listing]. Ther Umsc 2007; 64: 495-503

3 Reich-Weinberger S, Schmitz M, Öfner-Velano $D$. A new treatment of the "open abdomen" with controlled negative pressure and a new device. 21th Conference of the European Wound Management Association (EWMA) 2011, Brussels, Belgium

\section{Dr. med. Carl Meissner}

Assistenzarzt

Klinik für Allgemein- und

Viszeralchirurgie

Klinikum Magdeburg gGmbH

Birkenallee 34

39130 Magdeburg

carl.meissner@gmx.de

\section{Prof. Dr. med. Gerd Meissner} Chefarzt

Klinik für Allgemein-, Thoraxchirurgie, Proktologie und Ernährungsmedizin Carl-von-Basedow Klinikum

Saalekreis $\mathrm{GmbH}$

Vor dem Nebraer Tor 11 06268 Querfurt 